\title{
Prof. Drs. h. c. Franz Pirchner PhD
}

\section{Jahre}

Professor Franz Pirchner PhD, geboren am 07.01.1927, bei Freunden und in englischen Fachkreisen besser bekannt als „Franz“ ist aus vollem Herzen ein Tiroler Bürger. Dies muss man wissen, wenn man seinen Werdegang und seine Karriere richtig einschätzen und würdigen möchte.

Im Elternhaus in Imst war neben der Gastwirtschaft der Viehhandel die wichtigste Einnahmequelle und Prof. Pirchner hat so von Kindesbeinen an, die Einschätzung des Wertes eines Tieres mitbekommen. So war auch der Weg zum Genetiker an der Veterinärfakultät und an die Bodenkultur in Wien eine „natürliche“ Sache. Doch seinen speziellen genetischen Schliff bekam er an der Iowa State University in den USA, bei Prof. J. Lush, wo er 1957 seine PhD machte und auch die Grundlagen zu seinem bekannten im Paul Parey Verlag erschienenen Buch „Populationsgenetik in der Tierzucht“ legte. Da es kein einfaches Buch war, schrieben sogar Freunde einen Begleittext (siehe Gravert; Vorlesungsmanuskripte), doch hatte dieses Buch den wohl grössten Einfluss auf die Entwicklung der Tierzucht in Europa und der Nachkriegszeit.

Wie es heute kaum mehr für eine wissenschaftliche Karriere möglich wäre, hat Prof. Pirchner noch in der Tierzuchtpraxis seine ersten Dollars und Schillinge verdient. Als Leiter einer Versuchsanstalt in Wieselburg und später als Chefgenetiker der Geflügelzuchtfirma Heisdorf-Nelson in Kirkland USA konnte er Theorie und Praxis auf einen Nenner bringen.

1964 wurde er als Professor für Tierzucht an die Tierärztliche Fakultät der Universität Wien berufen.

Verschiedene Gründe veranlassten ihn 1970, den Ruf an den Lehrstuhl für Tierzucht der Technischen Universität München in Freising / Weihenstephan anzunehmen, sicher aber auch, da Tirol zu München näher lag als zu Wien.

Seine segensreiche Tätigkeit erstreckte sich nicht nur auf Lehre und Forschung an Universitäten, er wirkte auch in vielen deutschen und internationalen Tierzuchtvereinigungen in leitenden Positionen. Von 1966 bis 1972 war er Leiter der Genetik-Kom- 
mission der Europäischen Vereinigung für Tierzucht, von 1984 bis 1988 Vorsitzender der Deutschen Gesellschaft für Tierzuchtwissenschaften. Besonders wichtig für die Tierzucht war seine Rolle als Herausgeber der Zeitschrift für Tierzüchtung und Züchtungsbiologie (Journal; Animal Breeding and Genetics), an der er bis heute noch massgeblich tätig ist und auch noch selbst publiziert. Genannt sei auch sein Engagement im Redaktionskollegium des „Archiv für Tierzucht“, dessen langjähriges Mitglied er ist.

Seine Ausstrahlung auf die wissenschaftlichen Entwicklungen der Populationsgenetik ist einmalig und ohne Vergleich, auch über alle früheren und aktuellen Grenzen hinweg. Offen für jede wissenschaftliche Neuerung und kompetent in den Grundlagen der Tierzucht war er Motor der europäischen Entwicklung der Nutztierwissenschaften. Voller Ideen und motivierend für alle Jungwissenschaftler hat er sich besonders für die akademische Jugend eingesetzt. Unzählige Diplomarbeiten, über 50 Doktorarbeiten und mehrere Habilitationen hat er neben seiner eigenen publizistischen Tätigkeit betreut.

Sein Interesse galt nicht nur den biostatistischen Analysen, er setzte sich auch für die Biodiversität und seltene Rassen, besonders der „Tiroler“ ein und veranlasste viele Kollegen die Forschung darüber aufzunehmen.

1988 erhielt Prof. Pirchner seinen ersten Ehrendoktor der Universität Ghent, Belgien, dem im Jahr 2000 ein zweiter Ehrendoktor der ETH Zürich folgte. 1995 wurde Prof. Pirchner von der Deutschen Gesellschaft für Züchtungskunde mit der Hermann von Nathusius Medaille ausgezeichnet.

Es ist wohl auch seiner Bescheidenheit zuzuschreiben, dass er nicht noch viele andere Ehrungen erhielt, die er eigentlich auf Grund seines Wirkens und seiner persönlichen Integrität verdient hätte. Selbst nach seiner Emeritierung erhielt er noch viele ehrenvolle Einladungen zu Vorlesungen, z. B. von Japan und anderen Ländern.

Losgelöst von den früheren administrativen Notwendigkeiten, findet er heute die wissenschaftliche Freiheit sich mit seinen Lieblingsthemen auseinanderzusetzen. Die jugendliche Neugierde gepaart mit seinem enormen Wissen macht ihn heute noch zu einem sehr interessanten Gesprächspartner. Er ist auch der einzige Populationsgenetiker, der im Kopf die Standardabweichungen errechnet und entsprechend die Signifikanzen interpretieren kann.

Prof. Pirchner zu beschreiben gelingt nicht vollständig, wenn nicht seine liebe Frau mit einbezogen würde. Ihre Fürsorge für die täglichen Bedürfnisse eines Wissenschaftlers waren getragen von der Einsicht, dass man nur kreativ sein kann, wenn man sicher ist, dass die Alltagsprobleme durch andere gelöst werden. Sie hat ihm all diese Probleme abgenommen und zeigte immer Verständnis für neue Situationen, in die sich Prof. Pirchner immer einbeziehen liess.

Alle „Ehemaligen“ möchten sich daher bei Prof. Pirchner für sein Engagement in vielen Sachen bedanken und wir alle - Schüler, Doktoranden, Habilitierende, ehemalige Mitarbeiter und Mitarbeiterinnen gratulieren dem „Chef“ zum 75igsten ganz herzlich. 